\title{
Evaluating Nurse Data Navigation Training Program: A Quality Improvement Project
}

\author{
Annette HORTON ${ }^{\mathrm{a}, 1}$ and Shannon ROSER ${ }^{\mathrm{a}}$ \\ ${ }^{a}$ Rockhampton Hospital Central, Queensland Hospital Health Service, Australia
}

\begin{abstract}
Nursing ability to navigate data by sourcing, visualizing, and managing data provides for skill and insights development. Effectiveness of clinical, operational and strategic decision making is underpinned by sound understanding of the data. The object of this quality improvement project is to design, deliver and evaluate a nursing specific training program using spreadsheet software Excel to improve nurse competence and confidence in data navigation.
\end{abstract}

Keywords. Data navigation, nurse training, Novice-to-Expert nursing practice, clinical, decision support, nursing informatics

\section{Introduction}

As Queensland Health moves further towards a whole of state digital health environment there is an explosion of clinical data. Understanding and visualizing data provides clinicians with insights to aide clinical, operational and strategic decision making. Training that is practical and functional taking into consideration a nurse's responsibility for own continuing professional development and lifelong learning is important for success. An inability to source and access this type of training specific to nursing is problematic. Excel is used as it is the most commonly used data visualization system used worldwide.

\section{Method}

An Excel training program Nurs-E was designed locally based on Benner's novice to expert framework. This training is designed and delivered by nurses for nurses with each module building on from the previous one with successive complexity. The training program includes four modules delivered four weeks apart to facilitate practice and embed skills, knowledge and experiential learning. The end product for participants at completion of program is an interactive dashboard allowing for ability to create unique combination of charts, graphs, and infographics to meet individual nurse needs.

Classes are kept to a maximum of six participants and is co facilitated due to nurse participants variable computer literacy skills so "no nurse is left behind". Co facilitation acts as a barometer check providing feedback to lead facilitator during each session to slow down, go back over an activity and provides one to one participant support as required. Training evaluations are completed at end of each module and a program

\footnotetext{
${ }^{1}$ Corresponding Author, Annette Horton; E-mail: Annette.Horton@health.qld.gov.au.
} 
evaluation is completed online anonymously by participants eight weeks post completion of program.

\section{Results}

Kirkpatrick evaluation model is used to evaluate the effectiveness of the training program. Class evaluations demonstrate a mean average of Strongly Agree for relevant content, improved understanding, useful teaching strategies and sensitive to learners needs which reflects the program meeting Level 1 and 2 of Kirkpatrick model.

Behavioral change of nurse participants and organisational benefit is determined from the online participant survey conducted eight weeks post program completion.

\section{Conclusion}

Sourcing, visualizing and managing data underpins sound clinical decision making. There is nursing appetite for data navigation training. Informatic training programs designed specifically for nursing that is practical and functional improves nurse competency and confidence.

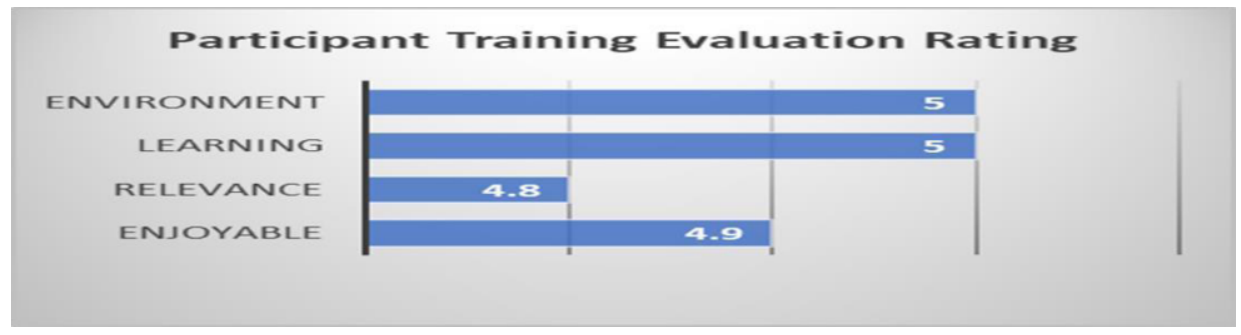

Figure 1. Participant training evaluations.

\section{References}

[1] Dorri S, Akbari M, Dorri Sedeh M. Kirkpatrick evaluation model for in-service training on cardiopulmonary resuscitation. Iranian journal of nursing and midwifery research, 21(5), (2016). 493497. doi: 10.4103/1735-9066.193396

[2] Reio Junior TG, Rocco TS, Smith DH, Chang E. A critique of Kirkpatrick's evaluation model. New Horizons in Adult Education \& Human Resource Development, 29(2), (2017), 35-53. doi: $10.1002 /$ nha3.20178

[3] Oh J, Shin-Jeong K, Sunghee K, Rajaguru V. Evaluation of the effects of flipped learning of a nursing informatics course. Journal of Nursing Education, 56(8), (2019), 477-488. doi: 10.3928/0148483420170712-06

[4] Courtney KL, Alexander GL, Demiris G. Information technology from novice to expert: Implementation implications. Journal of nursing management, 16(6), (2008),692-699. doi: 10.1111/j.13652834.2007.00829.x

[5] Queensland Audit Office. Digitising public hospitals report. 10:2018-19, (2018), Brisbane, Australia: Queensland Government. 